\title{
Proof of Beal Conjecture
}

\section{Zengyong Liang}

$\mathrm{MCHH}$ of Guangxi, Nanning, China

Email:1zy2ok@126.com

How to cite this paper: Liang, Z.Y. (2019) Proof of Beal Conjecture. Advances in Pure Mathematics, 9, 429-433. https://doi.org/10.4236/apm.2019.95021

Received: March 26, 2019

Accepted: May 19, 2019

Published: May 22, 2019

Copyright () 2019 by author(s) and Scientific Research Publishing Inc. This work is licensed under the Creative Commons Attribution International License (CC BY 4.0).

http://creativecommons.org/licenses/by/4.0/

\begin{abstract}
Beal conjecture is a famous world mathematical problem and was proposed by American banker Beal, so to solve it is more difficult than Fermat's last theorem. This paper uses relationship between the mathematical formula and corresponding graph, and by characteristics of graph, combined with the algebraic transformation and congruence theory of number theory; it is proved that the equation can only be formed under having a common factor and Beal conjecture is correct.
\end{abstract}

\section{Keywords}

Algebraic Formula, Graph, Algebraic Transformation, Congruence

\section{Introduction}

Beal conjecture is named after D Andrew Beal, after studying Fermat's last theorem in number theory. A spokesman for the American Mathematical Association, Bren, he said that to solve it is more difficult than another related mathematical problem, Fermat's Last Theorem [1] [2].

"No language proof" (for short PWWs) has been widely known in magazines published by the American Mathematics Association, especially in Mathematics Journal and Mathematics School Journal. Martin Gardner discussed PPWs. In his famous column Mathematics Games (1973) of an American scientific journal, Gardner states that "in many cases, dull proof can be supplemented by geometric figures, and it is so simple and beautiful that the truth of the theorem can be seen in an instant" [3], proving the most famous examples of indefinite equation by graphs; see (1) of Figure 1. The proof of Pythagoras theorem is given by Euclidean geometry's original 47th proposition [3] [4] [5]. Inspired by this, the author found an effective way to prove the Beal conjecture.

\section{Propositional Proof}

Proposition (Beal conjecture). If 


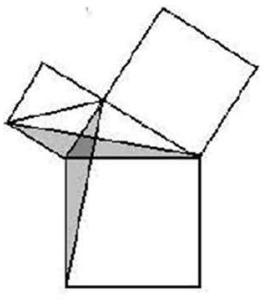

(1)

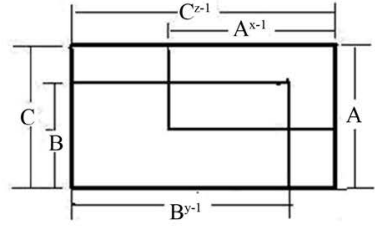

(2)

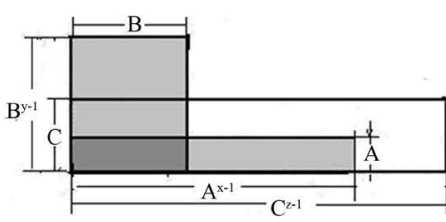

(3)

Figure 1. Three examples of proof $s$ by graphs.

$$
A^{x}+B^{y}=C^{z}
$$

where $A, B, C, x, y$ and $z$ are positive integers; and $x, y$ and $z$ are all greater than 2, then $A, B$ and $C$ must have a common prime factor [2].

Because $x, y$ and $z$ are larger than 2, firstly, the three powers in the Formula (1) are replaced by the areas of three rectangles that are $A A^{x-1}, B B^{y-1}, C C^{z-1}$, as (2) of Figure 1. If the Equation (1) is true, the area sum of rectangles $A A^{x-1}, B B^{y-1}$ should be equal to the area of rectangle of $C C^{x-1}$.

Theorem 1. We called both graphs is isomorphic if the relation of corresponding edges of three rectangles are the same. All isomorphic graphs are equivalent.

Proof. Because for two isomorphic graphs, the corresponding unknowns are the same in the formula, then the conclusion of the analysis should be the same.

From Theorem 1, we can discuss rectangles of different sizes and shapes with a representative figure, such as (3) Figure 1.

Next, we discuss this conjecture in two ways:

1) When $A, B$ and $C$ have no common factor

a) If $A=2 a, C=3 a, A^{x-1}=u B, B^{y-1}=(n+3) a, C^{z-1}=(u+1) B$, as (1) of Figure 2

Obviously,

$$
\begin{gathered}
A A^{x-1}+B B^{y-1}=2 a u B+(n+3) a B=(2 u+n+3) a B ; \\
C C^{z-1}=3 a(u+1) B,
\end{gathered}
$$

By (2) and (3), $A A^{x-1}+B B^{y-1} \neq C C^{z-1}$.

If $n=u$, then

$$
\begin{gathered}
A A^{x-1}+B B^{y-1}=(2 u+n+3) a B=3(n+1) a B \\
C C^{z-1}=3 a(u+1) B=3 a(n+1) B,
\end{gathered}
$$

By (4) and (5), $A A^{x-1}+B B^{y-1}=C C^{z-1}$, here, $A A^{x-1}, B B^{y-1}$ and $C C^{z-1}$ have common factor $a$.

b) If there $A^{\prime}, B^{\prime}, C^{\prime}$ have no common factor, as (2) of Figure 2, as

$$
\begin{gathered}
A^{\prime x-1}=n B+t ; \quad A A^{\prime x-1}=2 a(n B+t) ; B^{\prime y-1}=w+n a+3 a ; \\
B B^{\prime y-1}=(w+n a+3 a) B . \text { Then } \\
A A^{\prime x-1}+B B^{\prime y-1}=2 a(n B+t)+(w+n a+3 a) B=a[2(n B+t)+(n+3) B]+w B(6)
\end{gathered}
$$




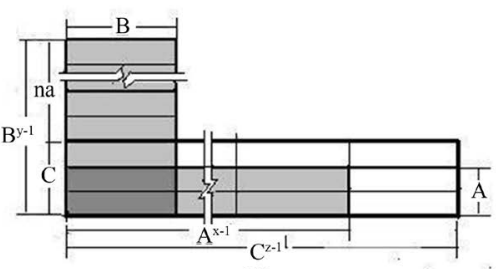

(1)

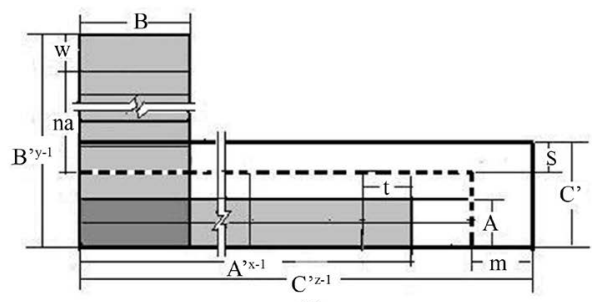

(2)

Figure 2. When $A, B, C$ have a common factor compared with no common factor.

and $C^{\prime}=3 a+s$, then

$$
C^{\prime z}=(3 a+s)^{z}=a\left[3^{z} a^{z-1}+z 3^{z-1} a^{z-2} s+\cdots+3 z s^{z-1}\right]+s^{z}
$$

By (6) and (7), according to the congruence theory [6], we seen that at least that $w B$ and $s$ have a common factor a, can be have

$$
A A^{\prime x-1}+B B^{\prime y-1}=C^{\prime z}
$$

In other words, the Equation (8) can only be established if $A, B$ and $C$ contain common factor $a$.

Sum up, we have been proved that the Equation (1) is not true when there is no common factor.

\section{2) When $A, B$ and $C$ have a common factor}

Look at the square in (1) of Figure 3. If $A=1$, then there is

$$
\begin{aligned}
& 1+8=9, \text { or } \\
& 1+2^{3}=3^{2} .
\end{aligned}
$$

If $A A$ and $C C$ are the products of two squares, and $C=3 A$, as (1) of Figure 3, then

$$
A A+8 A A=9 A A
$$

If $B B=8 A A, C C=9 A A$, then we obtain

$$
A A+B B=C C \text {. }
$$

It is say, when $A, B, C$ have common factor, then can write as (10) and (11).

When $A=D^{i}$, by substituting it into (10), we obtain as (2) of Figure 3, and

$$
D^{2 i}+8 D^{2 i}=9 D^{2 i}
$$

It can also be obtained by multiplying $D^{3 i}$ on both sides of (9), have

$$
D^{3 i}+2^{3} D^{3 i}=3^{2} D^{3 i} \text {. }
$$

Let $D=3$, by substituting it into (13), we obtain

$$
3^{3 i}+\left(2 \times 3^{i}\right)^{3}=3^{2+3 i}
$$

These are general term formulas for Beal conjecture. Let $A=3^{i}, B=2 \times 3^{i}$, $C=3, x=3, y=3, z=2+3 i$, by substituting it into (14), we obtain

$$
A^{x}+B^{y}=C^{z} \text {. }
$$

For example of (3) in Figure 3, we saw 9 cubes, the equation corresponding as below 


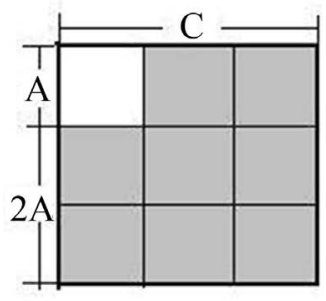

(1)

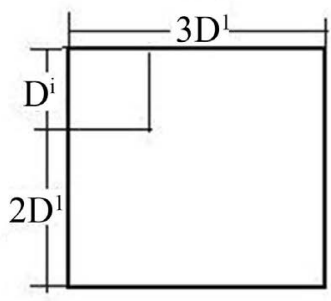

(2)

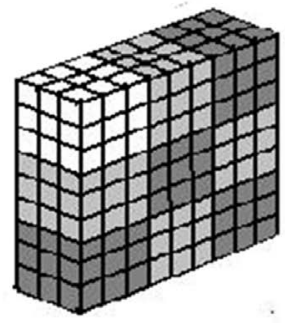

(3)

Figure 3. Three examples of graphs when edge length contains common factor.

$$
3^{3}+8 \times 3^{3}=9 \times 3^{3} \text {, or, } 3^{3}+6^{3}=3^{5} .
$$

For more examples as $9^{3}+18^{3}=3^{8}, 9^{6}+162^{3}=3^{14}, \cdots$.

Obviously, when $i$ is any integer, no matter the Equation (12) or Equation (14), they are all established.

Here, we noted that $A^{x}, B^{y}, C^{x}$ in (12) must be have a common factor $D^{2 i}$, and $D=3, i \equiv 0(\bmod 3)$; that in (13) must be have a factor $D^{3 i}$. The exponents are multiples of 3 , to merge with $2^{3}$. And the $D$ is 3 , to merge with $9 D^{2 i}$ form $3^{2+2 i}$. This is a necessary condition for the establishment of the equations of the Beal conjecture.

In addition, we have the second kind formulas:

$$
2^{i}+2^{i}=2^{i+1} .
$$

For example, $2^{12}+2^{12}=2^{13}, 4^{6}+8^{4}=2^{13}$.

Therefore, we proved that Equation (1) is maybe holds when A, B, C have a common factor.

In conclusion, we have been proved the Proposition, which Beal conjecture is true.

\section{Conclusion}

The above proves that, the Equation (1) is not valid without common factors; and it maybe holds when having common factor. It proved that, Beal conjecture is correct. Using the relationship between algebraic formulas and graphs and algebraic transformation, a world mathematical problem of indefinite equation is solved smoothly. It shows that sometimes general and geometric figures can make mathematical proofs simple and clear, which is indispensable. At the same time, this paper also finds a new and effective way to solve the indefinite equation in number theory.

\section{Conflicts of Interest}

The author declares no conflicts of interest regarding the publication of this paper.

\section{References}

[1] Baike.baidu Beal's Conjecture. https://baike.so.com/doc/7584609-7858703.html 
[2] AMS Beal Prise.

http://www.ams.org/profession/prizes-awards/ams-supported/beal-prize

[3] Nelsen, R.B. (2014) Mathematical Protrait-Proof Without Language. Translated by Xiao Zhankui and Fu Wenwen, Machinery Industry Press, Beijing, 1-71.

[4] Euclid (2011) Geometric Origin. Translated by Yan Xiaodong, Jiangsu People's Publishing House, Nanjing, 44.

[5] Xiao, W.Q. (2003) Mathematical Proof. Dalian University of Technology Press, Dalian, 43-44.

[6] Harly, G.H. and Wright, E.M. (2007) An Introduction to the Theorem of Number. People's Post and Telecommunications Press, Beijing, 48-57. 